\title{
Testimonianza per Gianfranco Folena
}

\author{
Piero Fiorelli
}

PUBBLICATO: 31 MAGGIO 2021

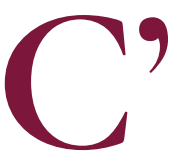

è in principio l'Arcadia. Non può non esserci. La storica Arcadia conosciuta come selvaggia regione pastorale nel cuore del Peloponneso; la poetica Arcadia rivissuta nell'egloga virgiliana, ripensata, tramandata col suo nome nei millenni; l'Arcadia infine che sul finire del Quattrocento un giovane poeta napoletano ha ricreato coi ricordi d'una fanciullezza pensosa, e che anni dopo, giusto alla svolta del secolo, ha cercato di render meglio accettabile a un pubblico fedele che, nel farsi via via più largo, vedeva pure accrescersi certe esigenze di forma. Non a caso i tre nomi dell'Ariosto, del Sannazzaro e del Bembo fronteggiano, sempre tra loro uniti, le Tre Corone trecentesche guidando la schiera degli autori moderni cosi nelle fonti dichiarate del primo nostro rimario (del Falco, I535) come in quelle del gran libro delle frasi toscane (del Montemerlo, I566). E sarà forse un caso, ma suggerito si può pensare da una freschezza di parole e di ritmi, trovare ammesso in raccolte di proverbi (dal Monosini in poi, I604) quel suo pensoso sorridere sul caso di chi "nell'onde solca e nell'arene semina" se "sue speranze funda in cor di femina".

Ma ecco. La precoce fortuna dell'Arcadia fra i testi esemplari della nostra letteratura volgare nel pieno Rinascimento non è dovuta solo alla grazia originale della sua scrittura; è dovuta in parte a una rilettura paziente dell'autore, pronto a rimediare ai guasti d'un'improvvida edizione dovuta ad altre mani e, insieme, a quei residui di provincialismo della stesura iniziale, almeno nelle parti in prosa, che non erano più accettati dal gusto letterario ormai dominante. Larte tipografica nata da poco imponeva, non foss'altro che per evitare correzioni e ricorrezioni e sperperi, un modello il più possibile unitario in fatto d'ortografia; la crescente circolazione dei libri a stampa in volgare favoriva un adeguamento a canoni linguistici accettati in tutta Italia; il pubblico dei lettori guardava con crescente rispetto ai modelli letterari del Trecento toscano, Dante Petrarca Boccaccio. Questi sono i segni d'una crisi linguistica italiana dell'ultimo Quattrocento. Il Sannazzaro e l'Arcadia sono nel centro, nel cuore stesso della crisi linguistica.

A quell'opera, a quell'autore e oggettivamente a quella crisi Gianfranco Folena dedica nel i946, sotto la guida di Bruno Migliorini, quella sua tèsi di laurea che la prigionia di guerra ha purtroppo ritardato: ma ci vedi a ogni pagina una familiarità coi testi, una padronanza dei metodi, una sicurezza di giudizio che non sono certo improvvisati nei pochi mesi d'una libertà ritrovata. E questo sia detto come in parentesi. In quella sua tèsi, in quel suo primo libro a cui la riduce nel 1952 per i tipi dell'editore Olschki, si può dire che si trovi concentrato un modo di vedere la nostra lingua, a pari titolo come oggetto di studio approfondito e come oggetto insieme di cura e di difesa, che io ricordo vivissimo nell'uno e nell'altro aspetto in quel Gianfranco Folena dei dieci o vent'anni in cui posso dire d'averlo conosciuto. E aggiungerò, ma in fondo è di troppo, una consonanza col pensiero e coll'agire pratico del comune maestro Bruno Migliorini. E diciamo ancóra, non vorrei qui dimenticare quello che immagino di vedere sullo sfondo, uno sguardo sorridente e incoraggiante di Giorgio Pasquali.

Una specie di nebbia mi sembra coprire i miei primi ricordi personali di Gianfranco. Il 1946 fu l'anno in cui frequentai più assiduamente il Migliorini del corso di lezioni e di Lingua nostra; ricordo che ebbe occasione proprio lui di presentarmi a un Fredi Chiappelli, a un Aldo Duro, anche a un Arrigo Castellani che pure avevo appena conosciuto al Circolo linguistico fiorentino. Ma a Gianfranco no, non ricordo; e anche nelle carte che si conservano del Circolo per gli ultimi anni '40 non pare che 
s'incontri il nome Folena, sì invece e spesso in quelle dei primi anni '5o (le ho potute vedere grazie al caro amico Parenti ultimo segretario), e invece il mio nome tutto il contrario: ma certo, è così, sarà dipeso da nostri spostamenti di sede, chi da Grosseto a Firenze, chi da Firenze a Roma.

Ah, perché mi perdo in questi particolari? Forse per un motivo: che in quegli anni della nostra prima conoscenza, in mezzo a comuni frequentazioni, più ancóra che il vólto dell'amico mi è rimasto impresso il suo nome in coppia fissa con quello del maestro, nei frontespizi di libri che dichiaravano una comune fedeltà: dell'uno all'altro e viceversa, e di tutt'e due a una lingua nazionale osservata di volta in volta come oggetto di studio storico nel suo progressivo formarsi a unità, e come oggetto d'un appassionato insegnamento inteso a far conoscere meglio quell'unità, a darle il suo senso più vero, a consolidarla. Due titoli gemelli: 1952, Testi non toscani del Trecento; 1953, Testi non toscani del Quattrocento: destinati alla ricerca universitaria: varietà regionali a confronto, che un giorno avranno finito col ridursi, è un fatto storico. E un titolo d'altro genere: 1954, Piccola guida di ortografia: destinato a dattilografe e tipografi: minute incertezze su come scrivere, che un giorno finiranno anche queste col ridursi, perché non crederci?

Ma negli stessi anni una volta il nome di Gianfranco è accostato a quello d'un altro e più lontano maestro: quando cura, I953, a pochi mesi dalla scomparsa di Giorgio Pasquali, l'edizione delle sue Conversazioni sulla nostra lingua, nate in gran parte per la radio, e cosi gradevoli anche a leggersi, tra le escursioni nei campi della storia e i richiami al perché di questa o quella norma o pretesa norma dell'oggi. Doppiamente gradevoli per un lettore fiorentino, che in quelle pagine d'un maestro romano e amantissimo della sua Roma trova a ogni passo un riconoscimento senz'ambagi della norma fiorentina di pronunzia, in anni in cui quelle e altre ambagi si vedevano ancóra suggerite dai resti d'un'infatuazione sociolinguistica per il mito della Capitale.

Un intervento di Bruno Migliorini in Lingua nostra, l'anno I949, vale ad avviare a soluzione un problema che nelle trasmissioni radiofoniche non può essere eluso, quello appunto d'una più attenta cura della pronunzia italiana da parte dei professionisti del microfono. E qualcosa comincia a muoversi, innanzi tutto nelle retrovie della lessicografia, dove sono da accertare e fissare le più esatte forme grafiche e foniche d'un alto numero di voci di lessico e di nomi propri, in italiano e in altre lingue, che quei professionisti non possono conoscere a memoria.

A questo comincia a provvedere fin da quello stesso anno, a Roma, il Dizionario enciclopedico italiano della Treccani, che si concluderà coi suoi dodici volumi l'anno ig6r e che ha nel Migliorini il soprintendente della parte lessicale. Vedremo: la cosa non finisce qui.

E qualcos'altro si muove nella R.A.I., che ha per allora il monopolio delle trasmissioni radiofoniche, appunto, e sta per estenderlo a quello delle trasmissioni televisive. Avendo l'occasione nel I952 d'aprire un corso d'addestramento per annunciatori della radio di nuova assunzione, la R.A.I. dispone che si svolga presso la sua sede di Firenze e incarica la coppia Migliorini-Folena di tenervi le lezioni di quella che vien denominata "fonetica", ma vuol essere propriamente "ortoepia"; spettano ad altri insegnanti le lezioni ed esercitazioni di dizione, le lezioni di lettura di quattro lingue straniere, gli ammaestramenti di cose più professionali e più tecniche. Non ho, non cerco documenti precisi, ma credo abbia avuto gran parte in queste novità un altissimo dirigente della radio che guardava lontano, il maestro Giulio Razzi.

Loccasione si ripete quattro anni dopo, in quella stessa sede e con quella stessa ripartizione delle materie d'insegnamento. Bruno Migliorini però, un po' per temporanee ragioni di salute e più per la crescente fatica del portare a compimento la sua grande Storia, non si sente più di tenere la sua parte 
di lezioni in prima persona: le lascia a un altro allievo, che, da lui stesso introdotto sette anni prima nell'officina lessicografica della Treccani, vi è stato incaricato, poco di poi, della cura d'una delle cose più nuove che il Dizionario enciclopedico venisse offrendo: le "trascrizioni fonetiche". Come potrei non ricordare quel pomeriggio, era per l'appunto il 2I aprile 1956, quando, andato a prendere alla stazione Gianfranco di ritorno da Padova, mi accompagnai con lui fino alla sede di Firenze della R.A.I. in Piazza, allora, di Santa Maria Maggiore, e da lui venni presentato all'ottimo e carissimo direttore l'ingegner Carlo Vigo? Cominciava così un lavoro comune, destinato di per sé a esaurirsi presto, alla metà di luglio, continuandosi però, o meglio ripetendosi in altre forme, in due direzioni diverse.

Alla coppia d'insegnanti cosi rinnovata, Folena-Fiorelli, spettano due mesi e mezzo di lezioni ed esercitazioni, che si tengono all'ultimo piano di Palazzo Strozzi, con un'alternanza che in fatto di giorni ha i suoi limiti obbligati negl'impegni d'insegnamento a Padova per l'uno e a Trieste per l'altro di noi, mentre in fatto di contenuti è regolata, solo di massima, dall'attribuzione all'uno (Folena) delle vocali e dell'accento, all'altro (Fiorelli) delle consonanti e del sistema. Le lezioni sono venticinque per ciascuno; e venticinque è, per combinazione, anche il numero degli allievi. Un po' meno di metà sono donne, un po' meno di metà vengono da Roma, un po' più di metà hanno già esperienze occasionali di lavoro da annunciatori, come si dice, a cachet; le loro età variano dai 2r o 22 anni ai 36; hanno tutti un titolo di scuola superiore, sei di loro una laurea. Mirano tutti a una carriera professionale nella radio, forse in futuro nella televisione, forse anche come giornalisti. Qualche nome da ricordare? Mi fo per me una domanda. Almeno uno, sì, mi rispondo: quello di Massimo Valentini, romano, dottore in legge, che si sarebbe fatto amare dal pubblico della tivvù per un esempio che dava di serietà e di stile e di simpatia, a cui certo non disdicevano una dizione e una pronunzia esemplari. Ed era accanto al suo microfono quando lo colse, ventott'anni dopo, morte improvvisa. Ma per non chiudere in tristezza ricordiamo pure, presente al corso del '56 come conduttrice di parte delle esercitazioni, quella che nel '24 (preistoria, a pensarci...) era stata la prima annunciatrice della nostra radio appena inaugurata, la buona Maria Luisa Boncompagni, credo anche lei romana.

Si, ricordo. Ma la memoria si giova pure di qualche appunto di mia mano, di qualche documento non ufficiale, comunque sia d'ufficio, che non ero piu sicuro d'avere in casa ma che alla fine ho ritrovato senza saper chi ringraziare. E qualcos'altro potrei pure aver da parte, fuori di qui; salvo che il confinamento in casa per difesa dal coronavirus non mi permette di muovermi cosi alla leggera. Vedremo.

Diciamo intanto che quei corsi d'addestramento o, come pure dicevano, di formazione professionale si sono ripetuti a intervalli irregolari altre quattro volte, nel '6o, nel '6r, nel '66, nel '68, tanto da dare, a qualche giornalista benevolo che ne ha scritto qualcosa, l'impressione d'una vera scuola in via di formazione; e qualcosa c'era davvero, nel quadro d'una "università della radio", che stando a certi progetti presto abbandonati avrebbe dovuto trovar posto nella nuova sede fiorentina della R.A.I, a Bellariva, in costruzione in quegli anni, inaugurata poi nel '68. Ma Gianfranco Folena non era più della partita: aveva a Padova la cattedra e un centro di studi che avrebbe reso illustre, la casa e la famiglia con tanti bei bambini che crescevano bene. A Firenze tocco a me d'interessarmi di quei corsi che si rinnovavano; il mio viaggiare settimanale da e per Trieste, che durò fino al '63, mi diede l'occasione di frequenti fermate di qualche ora a Padova, per rivederlo e parlare di quel che teneva ancóra vicini i nostri studi, anche per dare informazioni e chiedere consigli a proposito di quel lavoro chera stato comune e continuava senza la sua presenza fisica. Dal '6o in poi, ebbi la fortuna di poter fare a mezzo le prime due volte con Dino Pieraccioni, e a lui si aggiunsero con cómpiti variati, le ultime due, Gianni Papini e Arrigo Castellani: tutti amicissimi, e più ancóra amici della nostra lingua, della sua storia e del suo avvenire. Ma, appunto, quell'esperienza avviata dal grande Bruno e dal grande Gianfranco non poté avere un séguito; e chi d'altra parte l'ha continuata per tutti gli anni '6o, e non 
oltre, presumerebbe troppo di sé nel caso che volesse recitare un mea culpa.

La televisione, nel diffuso interesse d'un pubblico sempre più vasto, stava cominciando a soverchiare la radiodiffusione. Alle poche voci più o meno scelte della radio, a quelle certe voci fatte consapevoli d'un loro ufficio, stavano subentrando le molte voci della televisione occasionali e imprevedibili. La professione dell'annunciatore, dello speaker, sarebbe stata abbandonata di li a poco aprendosi al mestiere meno semplice, e più attraente, del giornalista o del conduttore. Infine la R.A.I. non avrebbe più conservato il monopolio che per mezzo secolo era stato suo, le sue voci avrebbero faticato a farsi sentire da tutti, a farsi sentire come l'offerta d'un modello, se ci fosse stato, di buona lingua.

Queste novità potevano bene esser prevedute da qualcuno, ottimista o pessimista che fosse. Ma parevano in tutti i modi una cosa ancóra lontana quando si aprì nel 1959, e durò per dieci anni interi, una collaborazione che mi legò di nuovo a Gianfranco Folena, e insieme con lui a tutta una cerchia di maestri più anziani, accademici della Crusca. In questo senso ho accennato prima a un lavoro comune che si sarebbe rinnovato anche in una direzione diversa. Si trattò del programmare e poi realizzare quello che si sarebbe chiamato il Dizionario d'ortografia e di pronunzia, il D.O.P., restando di nuovo nell'àmbito della R.A.I.

Si, fin dal '54 la R.A.I. s'era pure affidata a un comitato d'esperti tra i più qualificati, che rifacessero opportunamente migliorato il Prontuario del ' 39 oggetto di tante discussioni. Ma fino al ' 58 era stato fatto cosi poco, e quel poco senza nessun impegno, e con un continuo contrasto di tutti i commissari coll'unico di loro rimasto fermo a quel molto fumo e poco arrosto di vent'anni prima, che nell'ambiente romano della Treccani non parve neppure cosa sfacciata una proposta che qualcuno fece: d'estrarre dal Dizionario enciclopedico, quando fosse finito d'uscire, un volume che desse soltanto le intestazioni delle voci, nelle loro ortografie e trascrizioni fonetiche, da offrire per una coedizione alla R.A.I., s'intende dopo un'attenta rilettura e una riduzione di mole. Il presidente Aldo Ferrabino accolse la proposta con aperta simpatia, la girò formalmente all'ente radiofonico, tenne una o più riunioni coi dirigenti di questo; ma la loro risposta, dopo qualche tentennamento, fu negativa: la R.A.I. voleva far da sé, voleva riaffermare una propria dimensione culturale e una propria vocazione pedagogica.

E cosi fu, nella forma. Nella sostanza, il comitato ora confermato, dimesso il commissario di minoranza e inclusi invece il Folena e il Fiorelli, confermò senz'altro quella proposta sfacciata d'una filiazione con opportuni filtri dal Dizionario enciclopedico. Deliberata sùbito l'assegnazione d'una più diretta responsabilità di redattori a Migliorini, Tagliavini e Fiorelli, tre nomi che a volte si vedono citati come di veri autori, ma impropriamente, tutti quanti gli otto componenti del comitato presero parte nel primo anno o due alla discussione e fissazione di tutti i criteri, nei successivi otto o nove alla rilettura e integrazione e correzione dei vari giri di bozze. Quante pagine di bozze non mi son passate fra le mani, portando in margine i segni di calligrafie diverse, dosservazioni diverse da sommare tra loro o da conciliare... Ricordo tra i piu attenti anche per le forme grafiche gl'interventi di Gianfranco Folena; superati di numero, forse, solo da quelli dell'altro grande Gianfranco, il Contini.

Padova è poi rimasta fuori del mio giro d'orizzonte. Cosi, per ragioni oggettive, di cattedre in discipline diverse e in sedi diverse. Ma Padova e il pavano, Venezia e il veneziano, e tutta la storia culturale cosi d'Europa come del Mediterraneo osservata coll'occhio d'un linguista d'alto livello, sono rimaste per me oggetto d'una cordiale ammirazione per un vecchio amico di cui non riuscivo più a tenere il passo. È già un mio vanto l'aver collaborato con lui, e con Bruno Migliorini comune maestro, in qualche lontano tentativo di far conoscere meglio le buone tradizioni della nostra lingua; e forse, di mantenerle in vita. 


\section{Cita come:}

Piero Fiorelli, Testimonianza per Gianfranco Folena, "Italiano digitale", XVII, 2021/2 (aprile-giugno) DOI: $10.35948 / 2532-9006 / 2021.7531$

Copyright 2021 Accademia della Crusca

Pubblicato con licenza creative commons CC BY-NC-ND 